\section{The consent and counselling of patients for cataract surgery: a prospective audit}

D Cheung ${ }^{1}$ and S Sandramouli ${ }^{2}$

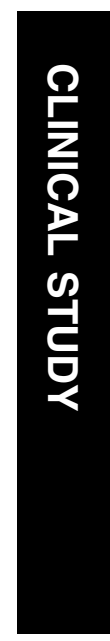

Purpose The aims of ideal preoperative patient adequately to enable an autonomous decision to be made without causing undue anxiety. We study how the paternalistic and nonpaternalistic approaches meet this ideal. The influence of the new patient consent forms is also assessed.

Methods Two cycles of a prospective clinical audit are presented. An assessment of relevant patient knowledge was performed by patient interview. Visual analogue scales were used to quantify patient anxiety.

Results The first cycle, examining a paternalistic approach, demonstrated: $37 \%$ of patients understood what a cataract was and $48 \%$ understood what surgery involved. $48 \%$ misunderstood that cataract surgery was completely risk free. In total, $80 \%$ of patients undergoing second eye surgery believed that it was completely risk-free. Average anxiety visual analogue scores (VAS) for cataract surgery were low (2.89). The second cycle, examining the nonpaternalistic approach combined with the implementation of new consent forms showed that, despite more explicit repeated preoperative consent: $39 \%$ of patients understood correctly what a cataract was, $28 \%$ understood what surgery involved and $43 \%$ misunderstood that surgery was completely risk-free. All patients undergoing second eye surgery thought that it was riskfree. The average anxiety VAS for cataract surgery were moderate (5.00).

Conclusions Both paternalistic and nonpaternalistic approaches to informed consent are inadequate in meeting the demands of the ideal informed consent. The new patient consent forms appear to have little effect in influencing patient knowledge about their surgery. Patients undergoing second eye surgery often have an overoptimistic view of cataract surgery.

Eye (2005) 19, 963-971. doi:10.1038/sj.eye.6701694; published online 15 April 2005

Keywords: cataract surgery; informed consent; medical ethics

\section{Introduction}

The concept of patient consent evolved from a judgement in the US Supreme court in $1914 .{ }^{1}$ Simple consent became part of international law following the World War II Nuremberg trial of Nazi physicians, ${ }^{2}$ with its basic premise that patient agreement is required prior to performing a procedure. The phrase 'informed consent' was first used in a 1957 California legal case $e^{3,4}$ and is defined as the process whereby health care providers provide the patient with the information necessary to make an informed decision about their care. By law, patients must consent to a procedure before it can be performed.

The benefits of informed patient consent for cataract surgery include educating the patient about the benefits of surgery and its associated risks. This in turn may lead to increased patient cooperation during surgery, thus minimising complications. For the surgeon/institution, a correctly completed consent form suggests that preoperative informed consent has occurred, increasing the chances of a successful defence against potential litigation. ${ }^{5}$ Despite these cited benefits, the consent process remains a topic of debate with some researchers stating that true autonomous informed consent can never occur due to a fundamental incompatibility between patient autonomy and physician responsibility. ${ }^{6}$

Theoretically, a signed consent form is considered valid until consent is withdrawn by
${ }^{1}$ Birmingham and Midland Eye Centre,

Birmingham, UK

${ }^{2}$ Wolverhampton and Midland Counties Eye Infirmary,

Wolverhampton, UK

Correspondence: D Cheung, Birmingham and Midland Eye Centre City Hospital, Dudley Road,

Birmingham B18 7QH, UK

Tel: +44 121554 3801;

Fax: + 447092275236 .

E-mail:dmwc@

bigfoot.com

Received: 2 May 2004

Accepted: 15 June 2004 Published online:

15 April 2005

Work based on two posters presented at Royal College of Ophthalmologists Congress, May 2004 No financial support. No proprietary interests. 
the patient. In practice, many hospital trusts, at the time of writing, had their own regulations regarding the duration of validity of a signed patient consent form, which was often arbitarily set at 6 months. The consent process was performed on the day of surgery; such to avoid its repetition since waiting times for surgery were often greater than 6 months. Some argue that informed consent would be more valid if it is obtained closer to the date of surgery, due to the effects of memory decay. ${ }^{7}$ Others contend that this is poor practice by not allowing the patient time to consider the management options fully. ${ }^{8}$ Regardless of timing, for consent to be considered valid, it must be implemented as a process and not just a single event and this is reflected in the opinion that the documentation of preoperative doctor-patient discussions about proposed management is seen as greater medicolegal defence than a consent form signed just prior to surgery. ${ }^{6}$

Recently, in seeking patients' consent: the ethical considerations, ${ }^{9}$ the GMC moved the UK medical profession closer to the American principle of informed consent. In the US, the law requires that patients need to be given a reasonable amount of information. In half the states, this is defined as the amount of information a reasonable physician would disclose. In the remaining states, this is defined as enough information a reasonable patient would require. ${ }^{6}$ The legal doctrine regarding informed consent is one based around the right of the patient (rights-based approach) to make his own decision about his/her future management with the doctor's role to provide the facts to enable this process, that is, patient autonomy. ${ }^{6,10}$ In contrast, the ethical idea of informed consent differs in that, although patient rights are still paramount, for example, right to refuse treatment; informed consent is viewed as a shared process of decision-making. Both patient and physician interact to evaluate the risks and benefits in relationship to the patient's situation. Finally, a shared decision is made based on this interaction. ${ }^{3,6,10}$ Shared decision-making therefore allows both patient autonomy and physician responsibility. How much each contributes to the shared process can vary and results in the different approaches to informed consent.

\section{The paternalistic approach}

Paternalism can be defined as a policy or practice of treating or governing people in a fatherly manner; by providing for their needs without giving them rights or responsibilities. ${ }^{11}$

Some authors believe that extensive preoperative counselling might have a detrimental effect in the process of informed consent by reducing a patient's ability to comprehend, ${ }^{5}$ increasing patient anxiety, ${ }^{5,12}$ and deterring some patients from proceeding with surgery. ${ }^{13,14}$ Indeed, it has been shown that many patients prefer to abstain from taking part in management decisions regarding their care. ${ }^{8}$ Thus some surgeons practise a paternalistic approach to the consent process; based on the belief that as long as the medicolegal requirements of valid informed consent have been met via previously performed documented preoperative counselling, further explicit counselling about the potential risks and complications, for the sake of obtaining a patient's signature on the consent form, should be avoided.

\section{Cycle 1-a paternalistic approach to informed consent}

\section{Aims}

The hypothetical gold standard/ideal approach to informed consent is one that adequately informs the patient about the forthcoming procedure to allow a reasoned decision without causing undue anxiety. At the time of commencement of this study, the preferred technique of one of the authors (DC) for obtaining informed consent was one based on the paternalistic approach. The aims of the first cycle of this audit were to discover how the paternalistic approach compared to this hypothetical ideal approach.

\section{Methods}

All consecutive patients undergoing cataract surgery by the same surgeon (DC) at the Wolverhampton and Midland Counties Eye Infirmary during the first 3 months of a 6-month specialist registrar rotation were recruited into the first cycle of this prospective clinical audit. The 3-month recruitment period was chosen to allow a second cycle to be performed in the same conditions. To achieve a relatively uniform study group of patients that would allow for later comparison, strict inclusion/exclusion criteria were imposed:

- The patient had to have undergone counselling about the nature of their condition, the details of the proposed operation, the benefits of surgery and the associated risks at the time of listing for their operation. There had to be formal documentation in their case records that this had occurred.

- They had to have read and understood a standard patient cataract information booklet ${ }^{15}$ that was given to all patients either at the time of listing for surgery or preassessment.

- The patient's first language had to be English.

- The operation had to be an uncomplicated local anaesthetic procedure. If complications did subsequently develop, the patient would be excluded from the study. 
- All patients under the age of 55 years were excluded. This was based on the authors' belief that younger patients were often more anxious.

- The patients had to have a normal psyche. That is, they were not suffering from depression, anxiety or a chronic confusional state.

- There was a normal fellow eye. That is, no 'single eyed' patients would be included since it was felt they would naturally be more anxious.

- If previous cataract surgery had been performed, then it had have been uncomplicated.
The patient was interviewed just prior to surgery and on the postoperative day with the aid of a data collection sheet (Figure 1).

The paternalistic approach

On the preoperative ward round:

- The patient was asked if they had any further questions about the surgery to be performed and whether they understood the risks and benefits.

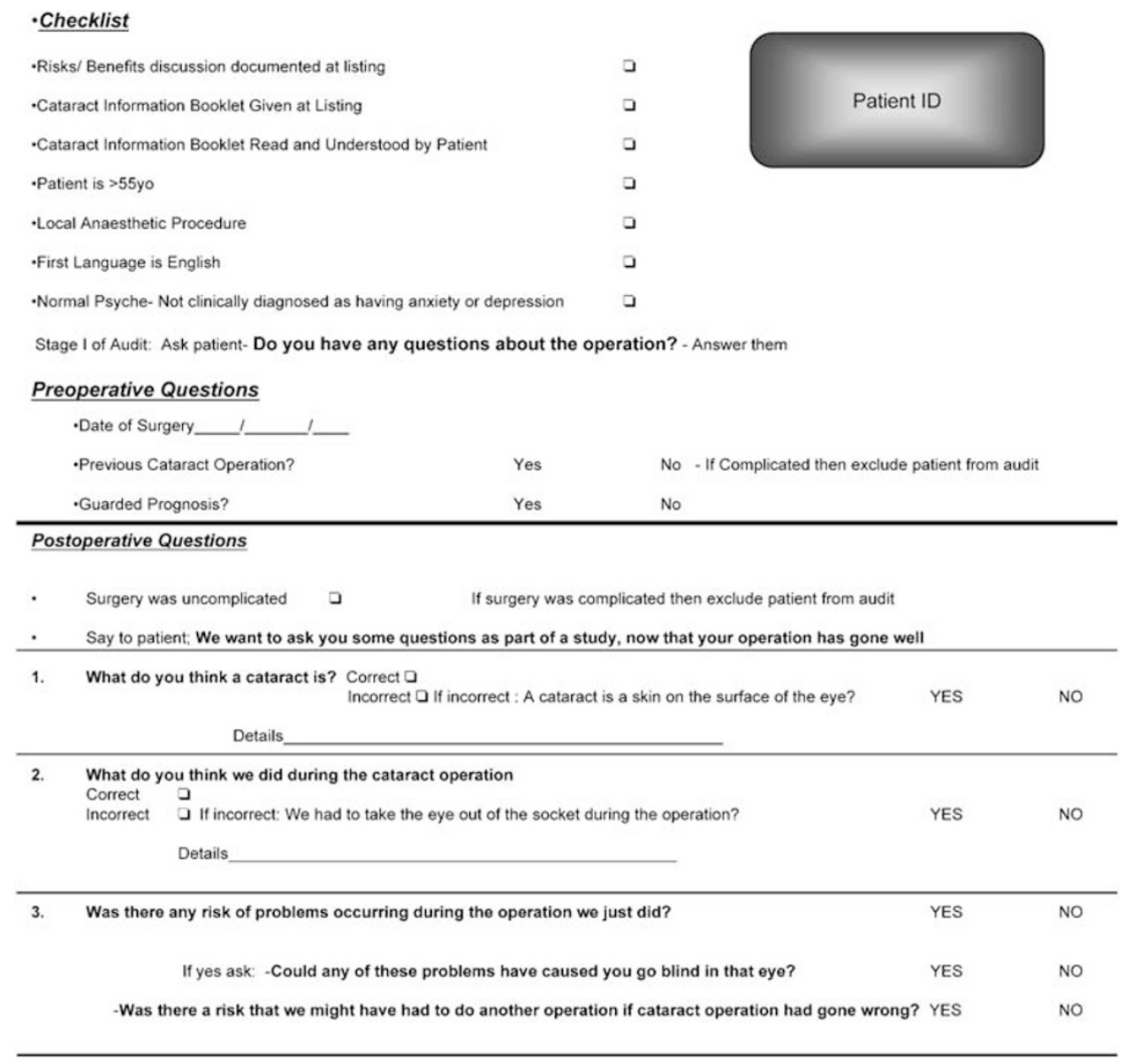

How anxious were you during this operation you've just had done?

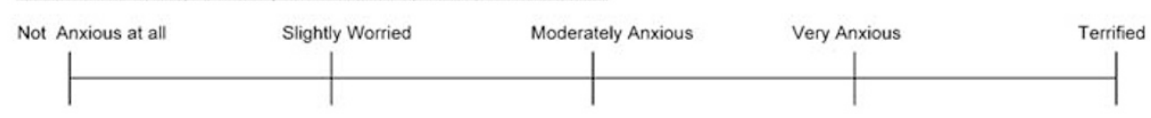

-How anxious are you when having dental surgery:

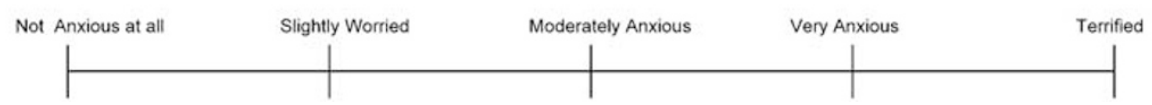

Figure 1 Data collection sheet. 
- If there were no further questions and the patient answered that they fully understood, the consent form would be signed and cataract surgery would then proceed.

- Any patient queries would be answered until the patient could positively acknowledge that he fully understood the risks and benefits of the proposed surgery. The consent form would then be completed and cataract surgery would proceed as normal.

- Despite not being explicitly reminded of the risks of surgery on the day of operation (unless requested by the patient); the acknowledgement by the patient that he/she had received enough information to allow them to make a decision to proceed toward surgery, constituted that the process of informed consent had occurred.

On the postoperative ward round the following morning, the patient was then interviewed about:

- Their basic understanding of what a cataract was and its surgical management.

- Whether or not there had been any risk of complications occurring and what these were.

- Their anxiety prior to and during surgery. This was graded using visual analogue scales (VAS).

- To enable interpatient comparability of patient anxiety, a control measurement of anxiety during a standard procedure, that is, dental surgery was also assessed.

- The questions were designed to be direct and often tested common public misconceptions about cataracts and their management.

- If the paternalistic approach to informed consent met the gold standard of ideal informed consent, the patient would have had adequate information to answer the questions correctly.

\section{Results}

\section{Patient recruitment}

From 1 July 2002 to 30 September 2002, 47 patients were personally operated on by the same surgeon (DC). Of these, 27 patients met the criteria for inclusion into the study. In all, 20 patients were excluded for the following reasons:

- One had complicated cataract surgery (vitreous loss following posterior capsular rupture).

- Ten because their first language was not English.

- Two were aged less than 55 years.

- Five had general anaesthesia.

- Two were 'only eyes'.

- Two had undergone previous complicated cataract surgery in their fellow eye.

\section{Demographics/past ocular history}

- There were five male and 22 female patients.

- The average age was 75 years. range: 61-93 years.

- Ten had undergone previous uncomplicated cataract surgery.

- Eight patients had been informed of a guarded visual prognosis for their operation-the main causes being ARMD, diabetic macular oedema, or glaucoma.

\section{Patient knowledge about cataracts, their management and the associated risks}

On the first postoperative day, the patients were asked:

What is a cataract?

- In all, 37\% (10/27) could describe correctly what a cataract was. All of these patients were undergoing cataract surgery for the first time.

- In all, 52\% (14/27) were incorrect in their description of a cataract.

- Of these, $71 \%(10 / 14)$ thought a cataract was a skin on the surface of the eye.

- One patient thought that it was calcium on the surface of the eye.

- Another patient thought it was an overgrowth of a third eyelid behind the eye.

- All patients undergoing second eye surgery were incorrect in their description of a cataract.

- The remaining $11 \%(3 / 27)$ had no ideas about what a cataract was.

Now that you have had the cataract operation, what do you think was done during the operation?

- Patients' descriptions of cataract surgery varied greatly. However, a response was deemed correct if the patient knew that the surgery was intraocular and involved surgical removal of the cataract, that is, not using lasers, with the implantation of an IOL.

- In all, $48 \%(13 / 27)$ of patients described correctly what cataract surgery involved. This included four of the $17(24 \%)$ patients undergoing first eye surgery and nine of the $10(90 \%)$ undergoing second eye surgery.

- In total, 33\% (9/27) of patients described the operation incorrectly:

- In all, 33\% (3/9) thought the eye was taken out of its socket.

$\circ$ A total of 22\% (2/9) thought the cataract was removed by laser surgery.

- A total of $11 \%$ (1/9) did not know an implant was put into the eye.

- Overall, 55\% (5/9) thought the cataract had to be scraped or peeled off the surface of the eye. 
- In total, $19 \%(5 / 27)$ of patients had no ideas about what cataract surgery entailed.

Your cataract operation has gone well without any problems. However, was there any risk of anything going wrong at any time?

- In total, 52\% (14/27) knew that there were possible risks involved in cataract surgery. However of these:

- In all, $42 \%(6 / 14)$ were unaware that ipsilateral blindness was a potential risk of cataract surgery (blindness unaware) while 58\% (8/14) were blindness aware.

- Overall, $14 \%(2 / 14)$ were unaware that there was a risk of requiring a second operation to rectify operative complications.

- A total of $48 \%(13 / 27)$ thought that cataract surgery was risk-free. This included $29 \%(5 / 17)$ of the patients undergoing first eye surgery and $80 \%$ $(8 / 10)$ of the patients undergoing second eye surgery.

\section{Anxiety VAS and the paternalistic approach to informed consent}

The patient was asked to mark on a linear scale (Figure 1):

- How anxious were you during the cataract operation just performed?

- How anxious would you have been, if instead of having a cataract operation, you were having dental extraction instead?

- By measuring the position of the mark on the scale, a visual analogue score (VAS) for anxiety was derived for each patient; where a score of 0 , meant the patient had no anxiety at all, to 10 , where the patient was as terrified as he/she could be.

\section{Average anxiety VAS for entire group}

- For cataract surgery: average anxiety VAS was 2.94 (sum of anxiety VAS for cataract surgery of all 27 patients /27); range 0-10.

- For dental extraction: average anxiety VAS was 4.97 (sum of anxiety VAS for dental extraction of all 27 patients /27); range 0-10.

\section{Subgroup analysis: anxiety VAS and its relationship with risk-awareness}

- For the 14 risk-aware patients:

- Average anxiety VAS for cataract surgery was 2.89 .

- Average anxiety VAS for dental extraction was 4.71 .

- Out of 14 risk-aware patients, the eight patients who were also blindness aware:
Average anxiety VAS for cataract surgery was 2.83.

Average anxiety VAS for dental extraction was 4.72 .

- For the remaining 13 risk-ignorant patients:

Average anxiety VAS for cataract surgery was 3.00.

Average anxiety VAS for dental extraction was 4.97.

\section{Subgroup analysis: anxiety VAS and its relationship with previous cataract surgery}

- For patients who were undergoing cataract surgery for the first time:

- Average anxiety VAS for cataract surgery was 3.03.

- Average anxiety VAS for dental extraction was 5.67.

- For patients who were undergoing cataract surgery for the second time:

Average anxiety VAS for cataract surgery was 2.80 .

- Average anxiety VAS for dental extraction was 3.85.

\section{Conclusions of first audit cycle}

Despite thorough preoperative counselling, the provision of patient literature and paternalistic informed consent, over a half of the patients did not know what a cataract was. In total, $11 \%$ are willing to undergo an operation for a condition they know nothing about. A total of $19 \%$ of patients having just had the operation knew nothing about what the procedure involved. Only $48 \%$ of the patients could correctly describe what cataract surgery involved and $48 \%$ of the patients thought that surgery was completely risk-free. These findings of poor patient comprehension are in keeping with other recent studies. ${ }^{8}$

The surgeon cannot assume that patients who have had previous cataract surgery to be more informed or less anxious than 'first eye' patients. None of the patients undergoing second eye surgery could give a correct description of a cataract; although they they were more aware that surgery was intraocular and required IOL implantation. Patients undergoing second eye surgery are just as anxious as patients undergoing surgery for the first time, with little difference between average anxiety VAS for cataract surgery for first (3.03) and second eye (2.80) patients. Hence, it is recommended that the counselling of patients must be to the same degree of explicitness for both patient groups.

Patients were consistently more anxious about having dental extraction than cataract surgery, despite the latter having potentially worse complications. Those patients who were aware of the risk of postoperative ipsilateral blindness showed similar average anxiety scores (2.83) to those who were unaware (3.00), suggesting that the practice of a paternalistic approach, to avoid repetitive explicit counselling in the hope of minimising patient anxiety, may be unfounded. In view of these findings and the possible medicolegal advantages incurred, a second 
cycle of this audit using nonpaternalistic/patient autonomy approach, with explicit/repetitive counselling was recommended.

\section{Cycle 2-a nonpaternalistic approach to informed consent}

\section{Introduction}

Nonpaternalistic/patient autonomy approach to informed consent

In a climate of increasing medical litigation, some clinicians have adopted a more defensive stance; that frequent explicit documented counselling of patients is necessary to minimise the risk of successful litigation against the doctor/trust should postoperative patient expectations be unmet. This non paternalistic/patient autonomy approach to informed consent is one based around the patient's rights. ${ }^{6}$ Here the role of the physician is to leave his own values aside and act purely as a provider of information for the patient. The patient assumes full responsibility for the decisions on his/her future treatment. Critics argue that a purely nonpaternalistic approach is irresponsible medicine because the doctor neglects his role as medical advisor; ${ }^{6}$ that '...compelling patients to receive information that they do not want or to make decisions that they do not wish to make is to fail to respect their dignity.' ${ }^{6}$ Indeed, some have commented that too much information may actually prevent the patient from making a reasoned autonomous decision. ${ }^{5,14}$

\section{New patient consent forms}

Following the inquiries into paediatric cardiac surgery at the Bristol Royal Infirmary $2001^{16}$ and the 1999-2000 inquiry into the removal, retention, and disposal of human tissue at the Royal Liverpool Children's Hospital at Alder Hey, ${ }^{17}$ Sir Liam Donaldson recommended a change to encourage patient trust in the consent process. In April 2002, five new consent forms were introduced, based on the recommendations of its Good Practice in Consent initiative advisory group. The new forms, which could be customised at a local level, encouraged patient consent to be more of a process than just a single event, with documentation of explicit counselling, both at the time of listing and on the day of surgery. The patient was given a copy of their signed consent form with contact details of the eye department so that any concerns about their forthcoming procedure could be discussed.

\section{Methods}

The second cycle of the audit therefore adopted the new consent form, since it appeared to address some of the recommendations of the first cycle. The period of study was lengthened to allow for recruitment of patients who had undergone informed consent using the new form. In our eye unit, it became mandatory for the clinic doctor to counsel and obtain patient consent for surgery using the first section of the consent form at the time of listing for surgery. The patient was given a carbon copy of their completed consent form for perusal. The operating surgeon was then obliged to repeat the process using the second part of the form on the preoperative ward round.

The eligibility/exclusion criteria were the same as the previous cycle; however, only patients who could confirm that they had received the patient copy of their consent form were eligible for inclusion. The measured outcomes were also identical.

The nonpaternalistic/patient autonomy-based approach to patient consent

On the preoperative ward round:

- The patient details were rechecked to ensure they met the criteria for inclusion into the study.

- They were then reminded about specific points about their imminent cataract surgery:

- There was a 1/100 chance of requiring a second operation to address a surgical complication.

- There was a 1/1000 chance of blindness in the operated eye following cataract surgery.

- Cataract surgery is not risk-free.

- That a cataract was an age-related opacification of the natural lens within the eye.

- That surgery involved removing the opaque jelly from the lens through a small incision into the eye and placing a plastic intraocular lens into the remaining capsular bag.

- The explanation was in such a manner as to make sure the patient could understand, aided by the use of models and cross-sectional diagrams of the eye.

- The patient was asked if he/she had any further queries about any aspect of the operation and any queries were answered.

- Once the patient could confirm that he/she understood all the potential risks, the consent form was again signed at the 'Confirmation of consent' section and the operation was performed.

\section{Results}

\section{Patient recruitment}

From October to January 2002, the same surgeon (DC) operated on 45 consecutive patients consented using the new process. Of these, 28 patients met the criteria for 
inclusion into the audit. The remaining 17 patients were excluded because:

- Nine of the patients did not speak English.

- Three patients underwent surgery under general anaesthesia.

- Four were 'only eye' patients.

- Two did not wish to partake in the study.

- Three were less than 55 years of age.

- Three suffered from dementia.

\section{Demographics/past ocular history}

- Ten male patients : 18 female patients.

- The average age was 80.7 years: range 74-90 years.

- Five patients had previously undergone uncomplicated cataract surgery to the fellow eye.

- Four had been informed about a possible adverse visual prognosis. The commonest causes being ARMD, myopic maculopathy, and glaucoma.

\section{Patient knowledge about cataracts, their management} and the associated risks

On the first postoperative day, the patients were asked:

What is a cataract?

- In all, 39\% (11/28) of patients could describe correctly what a cataract was.

- All of these patients were undergoing cataract surgery for the first time.

- In total, $46 \%(13 / 28)$ were incorrect in their descriptions of cataracts. Of these:

- Overall, 69\% (9/13) thought it was a skin on the surface of the eye.

- The remaining 31\% (4/13) thought it was a skin within the eye.

- All the patients undergoing second eye surgery were incorrect in their description of a cataract.

- A total of $14 \%(4 / 28)$ of patients did not have any idea what a cataract was.

Now that you have had the cataract operation, what do you think was done during the operation?

- In total, $28 \%(8 / 28)$ were able to describe correctly what cataract surgery involved. All of these were patients undergoing cataract surgery for the first time.

- Overall, 46\% (13/28) incorrectly described what cataract surgery involved.

- This included all five of the patients undergoing second eye surgery.
- Four patients thought that the eye had to be taken out of its socket.

- One patient thought that surgery involved peeling off an extra skin on the surface of the eye.

- One patient did not know that an IOL was inserted.

- In all, 25\% (7/28) of patients had no ideas about what cataract surgery entailed.

Your cataract operation has gone well without any problems. However, was there any risk of anything going wrong at any time?

- In total, $57 \%(16 / 28)$ patients were aware that there were risks involved in cataract surgery (risk aware). Of these:

- In all, 75\% (12/16) patients were aware that there was a risk of ipsilateral blindness following cataract surgery (blindness aware).

- Overall, 25\% (4/16) did not know that ipsilateral blindness was a potential risk following cataract surgery.

- All 16 patients (100\%) were aware that a second operation might have been required to address a complication of cataract surgery.

- A total of $43 \%(12 / 28)$ thought that cataract surgery was completely risk-free (risk ignorant).

Subgroup analysis: risk awareness and its relationship to previous cataract surgery

- Of the 23 patients undergoing cataract surgery for the first time: $30 \%(7 / 23)$ thought that it was risk-free.

- Of the five patients undergoing cataract surgery for the second time: $100 \%(5 / 5)$ thought that it was risk-free.

\section{Anxiety VAS and the nonpaternalistic/patient autonomy-based approach to informed consent}

\section{Average anxiety VAS for entire audit group}

- Average anxiety VAS for cataract surgery was 5. 00 (sum of all anxiety VAS for cataract surgery of all 28 patients /28): range 0-10.

- Average anxiety VAS for dental extraction was 6.42 (sum of all anxiety VAS for dental extraction of all 28 patients /28): range $0-10$.

Subgroup analysis: anxiety VAS and its variation with risk awareness

- For the 16 patients who were risk aware:

- Average anxiety VAS for cataract surgery was 5.31.

- Average anxiety VAS for dental extraction was 6.56. 
- For the 12 patients who were also blindness aware:

- Average anxiety VAS for cataract surgery was 4.58.

- Average anxiety VAS for dental extraction was 6.04 .

- For the 12 patients who were risk ignorant:

- Average anxiety VAS for cataract extraction was 5.41.

- Average anxiety VAS for dental extraction was 6.45.

\section{Subgroup analysis: anxiety VAS and its relationship with previous cataract surgery}

- For 23 patients undergoing cataract surgery for the first time:

- Average anxiety VAS for cataract extraction was 5.41 .

- Average anxiety VAS for dental extraction was 6.52.

- For five patients who were undergoing cataract surgery for the second time:

- Average anxiety VAS for cataract extraction was 2.50 .

- Average anxiety VAS for dental extraction was 5.00.

\section{Conclusions}

The time constraints imposed and strict recruitment criteria somewhat reduce the reliability of the results due to low patient numbers. However, despite this, some significant findings are apparent. Repetitive explicit counselling and the introduction of the new patient consent forms appeared to have little effect in improving patient performance on the questionnaires, with low proportions of patients able to describe correctly what a cataract was $(39 \%)$, what cataract surgery entailed $(28 \%$ correct) or whether there were any associated risks (57\% correct). As in the first cycle, patients undergoing cataract surgery to their second eye performed surprisingly poorly in comparison with patients undergoing first eye surgery.

One criticism of this study is that the use of patient questionnaires may be flawed since it may be a greater test of recall than understanding. Patient recall and understanding do not necessarily correlate. As Miesel and Kuczewski stated, 'While it might be true that some who cannot retain information for a few seconds might not be said to understand it, people often make reasonable decisions but cannot recall the premises that supported the reasoning or process that led to the conclusion. Nevertheless, they might well have understood it at the time. ${ }^{6}$

Again, patients were more anxious about having dental extraction surgery than cataract surgery. However, in this second cycle of the audit, the patients were also more anxious about cataract surgery than in the first cycle (average anxiety VAS for cataract extraction in first cycle $2.95 v s$ average anxiety VAS for cataract surgery in second cycle 5.00). Whether or not this is due to the effect of the extra counselling about the potential risks and the use of the new patient consent form is unknown. This group of patients studied may have been an inherently more anxious group anyway as shown by higher average anxiety scores for dental extraction (average anxiety VAS for dental extraction in first cycle $4.97 v s$ average anxiety VAS for dental extraction in second cycle 6.42).

Both cycles of this audit demonstrate the resistance encountered in educating patients about their condition and its treatment. The factors involved in improving patient knowledge about their condition and its treatment appear to be more complex than just increasing the amount of information given. ${ }^{10}$ These findings question whether true autonomous patient consent can ever be achieved since our ability to educate our patients adequately appears impossible. This has led some researchers to accept that although full comprehension by patients is often absent during the consent process, adequate comprehension may be present and considered suffice for the informed consent to be valid. ${ }^{18,19}$ Despite increased pressures for clinicians to practise a patientrights-based approach to informed consent, it was apparent that a significant proportion of patients demonstrated a reluctance/inability to partake in the shared decisions relating to their management; a similar finding to previous studies. ${ }^{8,20}$

It is difficult to produce any recommendations from this audit about the ideal frequency, timing and explicitness of patient counselling since some of the findings may be due to the poor statistical power of the small patient numbers involved in each cycle. Repeating both cycles of this audit with larger patient numbers in each cycle would help resolve some of these issues, as would the introduction of quantitative analysis to study the influences of frequency of counselling, explicitness of counselling and other modalities of patient information, for example, patient information videos. The two cycles of this audit suggest that neither the paternalistic nor the nonpaternalistic approaches to informed consent described are ideal in meeting the challenges of achieving true valid consent.

\section{References}

1 Nick WV. Informed consent-the new decisions. Bull Am Coll Surg 1974; 59: 12-17.

2 Nuremberg Military Tribunal. The Nuremberg Code. JAMA 1996; 276: 1691.

3 Katz J. The Silent World of Doctor and Patient. Free Press, Macmillan Inc.: New York, 1984. 
4 Salgo vs. Leland Stanford Jr University Board of Trustees. 154 Cal App2d 560,317 P2d 170 (1957).

5 Epstein LC, Lasagna L. Obtaining informed consent: form or substance. Arch Intern Med 1969; 123: 682-688.

6 Seeking Patient's Consent: The Ethical Considerations. General Medical Council publication: London, 1988.

7 Meisel A, Kuczewski M. Legal and ethical myths about informed consent. Arch Intern Med 1996; 156: 2521-2526.

8 Kiss CG, Richter-Mueksch S, Stifter E, Diendorfer-Radner G, Velikay-Parel M, Radner W. Informed consent and decision making by cataract patients. Arch Ophthalmol 2004; 122: 94-98.

9 Scanlan D, Siddiqui F, Perry G, Hutnik CML. Informed consent for cataract surgery: what patients do and do not understand. J Cataract Refract Surg 2003; 29: 1904-1911.

10 Lidz CW, Appelbaum PS, Miesel A. Two models of implementing informed consent. Arch Intern Med 1988; 148 1385-1389.

11 American Heritage Dictionary of the English Language, 4th edn. Houghton Mifflin Press: New York, 1990.

12 Faden RR, Beauchamp TL. A History and Theory of Informed Consent. Oxford University Press Inc.: New York, NY, 1986.
13 Katz RL. Informed consent: is it bad medicine? West I Med 1977; 126: 426-428.

14 Patten BM, Stump W. Death related to informed consent. Tex Med 1978; 78: 49-50.

15 Understanding Cataracts. RNIB and Royal College of Ophthalmologists Information Booklet for Patients. The Royal College of Ophthalmologists: London, 2001.

16 Kennedy I. Learning from Bristol: The Report of the Public Inquiry into children's heart surgery at the Bristol Royal Infirmary 1984-1995. The Stationery Office Ltd: London, 2001.

17 Redfern M, Keeling JW, Powell E. The Royal Liverpool Children's Inquiry: Report (House of Commons Paper) 2001. The Stationery Office Ltd: London, 2001.

18 Sreenivasan G. Does informed consent to research require comprehension? Lancet 2003; 362: 2016-2018.

19 Levine RJ. Ethics and Regulation of Clinical Research, 2nd ed. Yale University Press: New Haven, 1988, pp 95-153.

20 Ingelfinger FJ. Informed (but uneducated) consent. $N$ Eng J Med 1969; 287(9): 465-466. 